\title{
India: Quality assurance procedure monitors and improves quality of services
}

Population Council

Follow this and additional works at: https://knowledgecommons.popcouncil.org/departments_sbsr-rh

Part of the Demography, Population, and Ecology Commons, Family, Life Course, and Society Commons, Health Services Research Commons, International Public Health Commons, and the Medicine and Health Commons

How does access to this work benefit you? Let us know!

\section{Recommended Citation}

"India: Quality assurance procedure monitors and improves quality of services," FRONTIERS OR Summary no. 76. Washington, DC: Population Council, 2008. 
India

Quality of Care

OR Summary 76

\section{Quality Assurance Procedure Monitors and Improves Quality of Services}

A quality assurance $(Q A)$ procedure was pilot-tested and subsequently scaled-up throughout the entire state of Gujarat, India after demonstrating that the checklist is an efficient tool for identifying and remedying gaps in service delivery. The $Q A$ procedure resulted in significant improvements in facility readiness and some aspects of service quality. The procedure is now being introduced in six more states.

\section{Background}

From 2004 to 2006, the Frontiers in Reproductive Health Program (FRONTIERS) collaborated with UNFPA and Indian state health officials to develop and test a standardized quality assurance (QA) procedure. The State Ministry of Health and Family Welfare (MOHFW) used the QA procedure and materials, which included tools, checklists, and a procedural manual, to assess health care services in rural clinics in two pilot districts each in the states of Gujarat and Maharashtra. Based on positive results, the State MOHFW expanded the QA procedure in a phased manner throughout all 25 districts of Gujarat. The model is now being introduced in six further states with support from USAID, GTZ and UNFPA; the Population Council is providing technical assistance with the expansion into Karnataka and Maharashtra states.

In the QA procedure, the MOHFW selects district QA teams of two or three district-level officials. The teams conduct three quarterly visits to each facility (including primary health centers or PHCs, community health centers or CHCs, and sub-centers). During each two- to three-hour visit, the QA team uses a 65-item checklist to assess and grade (A through D) each facility in terms of infrastructure, staff training and technical competence, supplies, procedures, interactions with clients, and services delivered, including family planning, maternal care, and detection and treatment of sexually transmitted infections.

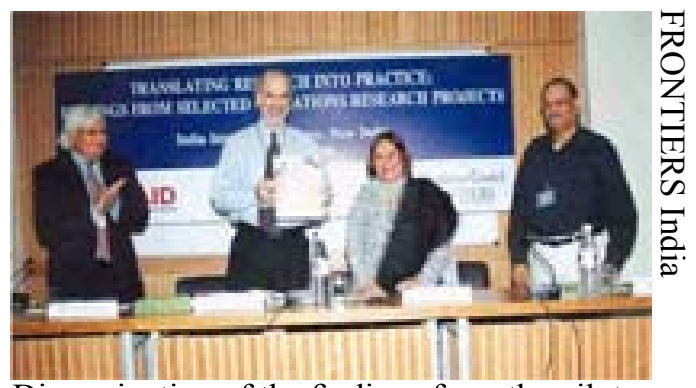

Dissemination of the findings from the pilot QA study led to expansion and replication.

The team suggests measures to address any gaps identified. The team uses the same tool to assess progress made in improving services during subsequent visits at three- to four-month intervals.

\section{Findings from pilot projects}

- Findings in the pilot facilities (16 PHCs and 6 CHCs) showed that the QA approach significantly improved facility readiness and quality of care. Most facilities received an initial overall rating of B or below. By the third quarterly visit, the grades of the majority of facilities had increased to $\mathrm{A}$, and only one facility scored a $\mathrm{C}$ grade.

\section{- Scores on selected indicators increased} significantly during the follow-up visits. In Dahod district, for example, the number of facilities providing at least three family planning methods increased from 35 percent to 100 percent by the third QA visit. However, improvements did not occur on all indicators. Even at the third visit, less than half of the facilities had a staff member trained in emergency obstetric care. 
- Based on the evidence from the pilot phase, the state government of Gujarat decided to incorporate the QA procedure into its Reproductive and Child Health program. FRONTIERS provided technical assistance in a phased scale-up of the intervention to all 25 districts. The first phase of the scale-up included 401 PHCs and 65 CHCs, about 35 percent of PHCs and CHCs in Gujarat.

- A total of 1,922 health care officials, including medical officers, regional and state health officers, QA tem members and leaders, and medical officers, received one- and two-day training sessions on conducting QA activities. The MOHFW appointed a state-level QA Coordinator to monitor the QA procedure and follow up within the districts.

- The checklists were successfully incorporated into routine monitoring systems. Key items requiring action in most facilities included facility infrastructure and cleanliness, availability of protocols and job aids, and maintenance of service records and reports.

- The QA procedure clearly showed progress or lack of progress. The proportion of facilities graded A on infrastructure and staff training doubled (from $17 \%$ to $34 \%$ ) from the first round to the second. However, scores on quality of care remained uniformly low - about one-fourth graded $\mathrm{C}$ and three-fourths graded D in both rounds.

\section{Expansion into new states}

- In Kenya, integration of the MIP within the existing health system strengthened sustainability. Strong community awareness of the IPT program helped to mobilize community anti-malaria ac- tions, such as re-treatment of bednets, to prevent malaria. Kenya's participation in regional antimalaria initiatives, such as Rollback Malaria and the Abuja Declaration, helped to position IPT as a national priority.

- In Malawi, the $\mathrm{MOH}$ prioritized malaria as a national problem and committed to a systematic, long-term search for solutions, including use of evidence-based interventions, development of simplified MIP guidelines for providers and the public, and a target of eliminating malaria as a public health problem within 20 to 30 years.

- This commitment to a specific goal and to an evidence-based approach elicited high levels of support from development partners. Strong links within the $\mathrm{MOH}$ and with district- and community-level organizations also enhance the potential for sustainability.

\section{Programmatic Implications}

- Monitoring quality through repeated visits to a facility and use of the QA checklist to rate readiness and service procedures is easily institutionalized within an existing supervisory system that is already functioning adequately. Enabling existing district-level supervisors to take on a quality assurance function is feasible. For maximum impact, however, the QA procedure must deployed over the long term (with strong support from senior management), incorporated into routine supervisory responsibilities, and facilitated with tools such as the QA checklist and manual.

July 2008

Sources: Khan, M.E., Anurag Mishra, Vivek Sharma, and Leila Caleb Varkey. 2008. 'Development of quality assurance in reproductive health services for district public health systems: Implementation and scale-up in the state of Gujarat," FRONTIERS Final Report. Washington, DC: Population Council. See Also: Khan, M.E. et al. 2008.'Institutionalization of quality assurance within district health management: Experiences from Maharashtra and Karnataka," FRONTIERS Final Report. Available on our website at www.popcouncil.org/frontiers or by e-mail: publications@popcouncil.org.

This publication is made possible by the generous support of the American people through the President's Emergency Plan for AIDS Relief (PEPFAR) and the United States Agency for International Development (USAID) under the terms of Cooperative Agreement No. HRN-A-00-98-00012-00. The contents are the responsibility of the FRONTIERS Program and do not necessarily reflect the views of USAID or the United States Government.

\section{Population Council}

